\title{
Fabrication and Analytical Applications of Hybrid Mesoporous Membranes
}

\author{
Akira Yamaguchi*,**† and Norio TeramaE* \\ *Department of Chemistry, Graduate School of Science, Tohoku University, \\ Aoba, Sendai 980-8578, Japan \\ **PRESTO, Japan Science and Technology Agency, 4-1-8 Honcho, Kawaguchi, Saitama 332-0012, Japan
}

\begin{abstract}
Recently, a hybrid mesoporous membrane composed of surfactant-templated mesoporous silica inside a porous anodic alumina membrane has been developed. Since this membrane allows the use of columnar silica-mesopores (silicananochannels) as nanofluidic channels, separation of molecules can be realized by mass transport through the silicananochannel with molecular dimensions. Here, we review the methods to fabricate the hybrid mesoporous membranes, their structural features, and the analytical applications of hybrid mesoporous membranes.
\end{abstract}

(Received September 1, 2007; Accepted September 26, 2007; Published January 10, 2008)

$\begin{array}{llll}1 \text { Introduction } & 25 & 4 \text { Analytical Applications } & 28 \\ 2 \text { Fabrication Methods } & 26 & 5 \text { Summary } & 29 \\ 3 \text { Mesostructures } & 27 & 6 \text { References } & 29\end{array}$

\section{Introduction}

Surfactant-templated mesoporous silica has inherent properties such as high surface area and adsorption capacity, uniform pore diameter ranging from a few to tens of nanometers, and a highly ordered pore structure. These properties have attracted much attention to the potential use of mesoporous silica for several analytical purposes. The early reports on the synthesis of mesoporous silica were concerned with hydrothermal synthesis of mesoporous silica powders with a few to tens of nanometers in pore diameter by using alkylammonium surfactants as a structural directing reagent. ${ }^{1-4}$ In the first analytical application of the mesoporous silica powder, the properties of high surface area and adsorption capacity were paid attention to develop an efficient sorbent and the efficient sorption of mercury ion was demonstrated in 1997. ${ }^{5}$ Since then, various studies have been performed to develop excellent sorbents based on the mesoporous silica materials with functional organic layers. ${ }^{6}$ Size-selective adsorption of proteins on mesoporous silica powders by utilizing the uniform pore diameter was demonstrated in 2001; mesoporous silica with $5.6 \mathrm{~nm}$ in pore diameter showed size-selective adsorption of proteins with smaller sizes than the pore diameter. ${ }^{8}$ In addition, some studies have been reported to develop a molecular recognition system by incorporating fluorophores within the mesoporous silica. ${ }^{7}$

On the other hand, some attempts have been carried out to use mesoporous silica powders as stationary phases in several chropatographic systems: capillary gas chromatography, ${ }^{9}$ high-

† To whom correspondence should be addressed.

E-mail: yakira@mail.tains.tohoku.ac.jp performance liquid chromatography (HPLC), ${ }^{10,11}$ and sizeexclusion chromatography (SEC). ${ }^{12}$ These chromatographic applications are based on expectations that the high surface area and the uniform pore diameter of the mesoporous silica should improve properties of both size exclusion and surface interaction in separation of molecules. ${ }^{12}$

Applications of mesoporous silica powders mentioned above are generally based on adsorption/desorption or distribution phenomena. In contrast, one of the interesting structural features of the mesoporous silica is one-dimensional (1D) alignment of columnar pores (silica-nanochannels) and the use of the 1D silica-nanochannel as a nanofluidic channel for separation and sensing has been expected. The nanofluidic systems based on mesoporous silica could be achieved by forming mesoporous silica thin film on a substrate. For example, Yamada et al. ${ }^{13}$ formed mesoporous silica with a $3 \mathrm{D}$ cubic mesostructure on a sensor substrate and sensing of NO gas transported through the silica mesopores was demonstrated. Liu et al. ${ }^{14}$ formed an azobenzene-modified mesoporous silica thin film with 3D cubic mesostructure on an ITO electrode and the conformation (trans or cis) of azobenzene regulated the effective pore size and the mass transport behavior of ferrocene derivatives. In spite of these studies, there is a possibility that lateral diffusion of molecules along the substrate prevents regulation of mass transport across the film containing $3 \mathrm{D}$ cubic mesoporous silica. Hence, it is highly desired to synthesize a mesoporous silica thin film with perpendicularly oriented 1D silica-nanochannels, by which mass transport across the film can be easily attained. Although an evaporation inducedselfassembly (EISA) method has been generally used to synthesize the mesoporous silica thin film composed of 1D silica nanochannels, ${ }^{15}$ the resulting film is composed of $1 \mathrm{D}$ 


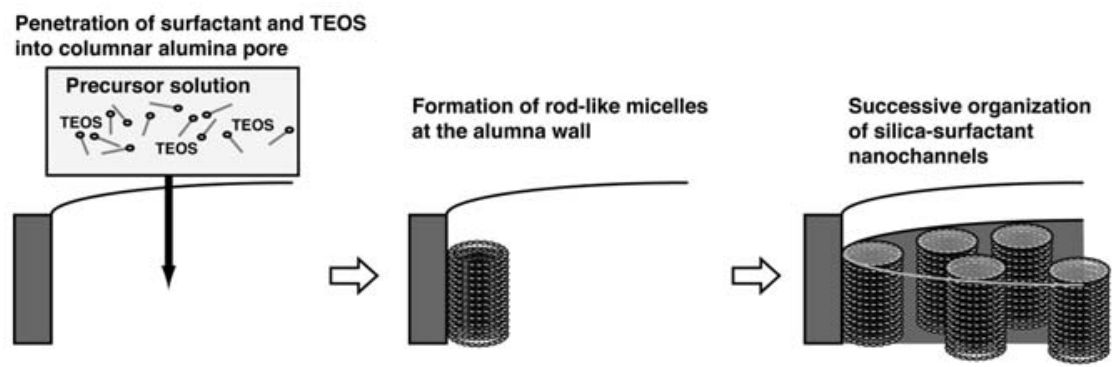

Fig. 1 Proposed scheme for formation of silica-surfactant nanochannels inside a columnar alumina pore.
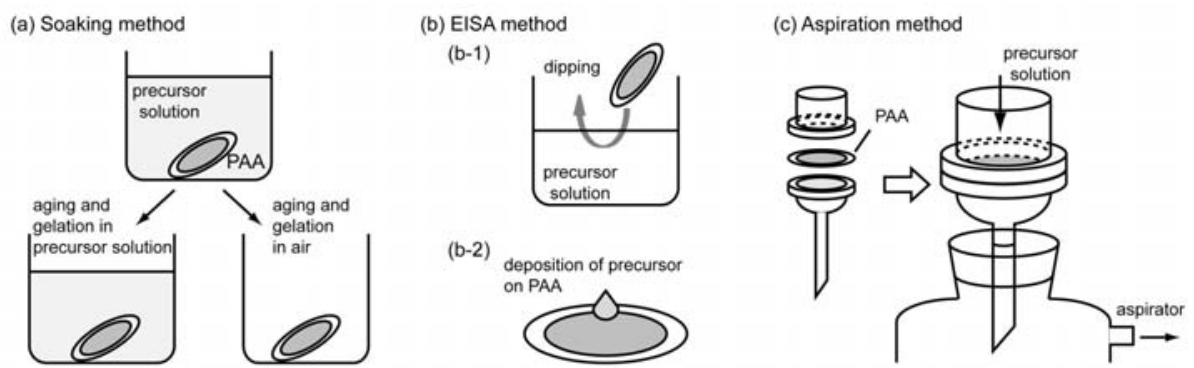

Fig. 2 Methods to fabricate hybrid mesoporous membranes.

silica-nanochannels oriented parallel to the surface of a planar substrate and this parallel orientation does not allow molecules to transport across the film. An attempt was made to achieve the perpendicular orientation by applying a high magnetic field, but the regulation of the orientation of silica-nanochannels was not complete. ${ }^{16}$ Therefore, the achievement of the perpendicular orientation had been a crucial problem in the synthesis of a mesoporous silica thin film.

Formation of mesoporous silica within a porous anodic alumina (PAA) membrane is a novel method for the use of mesoporous silica as the 1D nanofluidic channel. The PAA membrane has a packed array of columnar alumina pores oriented perpendicularly to the membrane surface. ${ }^{17}$ Since the formation of mesoporous silica inside the columnar alumina pore sometimes gives 1D alignment of silica-nanochannels along the columnar alumina wall, the resulting silicananochannels are predominantly oriented perpendicularly to the membrane surface. ${ }^{18}$ Hereafter, the PAA membrane with mesoporous silica is called a hybrid mesoporous membrane. Since the hybrid mesoporous membrane allows mass transport through the silica-nanochannels within the membrane by embedding it between two solution phases, it can be simply applied for separation of molecules and sensors by utilizing mass transport through 1D silica-nanochannel. Thus, the hybrid mesoporous membrane has a capability for various analytical systems. Here, we review the methods for the fabrication of hybrid mesoporous membranes, and their structural features, as well as applications of the hybrid mesoporous membrane for analytical purposes.

\section{Fabrication Methods}

The fabrication of the hybrid mesoporous membrane essentially relies on the self-organization of silica-surfactant nanocomposites at a solid/liquid interface like formation of the mesoporous silica thin films. ${ }^{15}$ When a precursor solution, containing a silica source (tetraethoxysilane (TEOS) or tetramethoxysilane (TMOS)) and a structural directing reagent (surfactant), is introduced inside the columnar alumina pores, the silica-surfactant nanocomposite grow inside the alumina pores. The growth of the silica-surfactant nanocomposite is considered to commence at the inner walls of the alumina pore (Fig. 1). ${ }^{18}$

The methods to fabricate the hybrid mesoporous membranes are roughly classified into three types (Fig 2). The first type involves soaking an alumina membrane in a precursor solution; this is based on a simple sol-gel method, in which a porous alumina membrane is put in a sol solution and the sol that infiltrates the columnar alumina pores turns to a gel during the drying process at an ambient condition or in vacuo (Fig. 2(a)). ${ }^{19-24}$ The second type includes an evaporation inducedself-assembly (EISA) method in which a precursor solution is loaded in the columnar alumina pores and mesoporous silica is grown during solvent evaporation (Fig. 2(b)). ${ }^{25-29}$

On the other hand, Yamaguchi et al. ${ }^{18,30}$ have proposed another method whereby an alumina membrane is set in an ordinary membrane filtration apparatus and a precursor solution is introduced into the columnar alumina pores under moderate aspiration (Fig. 2(c)). ${ }^{2}$ Since the mesoporous silica is grown when the precursor solution passes through the columnar alumina pores, hardly any formation of mesoporous silica occurs on the membrane surface.

The former two methods allow formation of columnar (cylindrical) mesoporous silica with well-ordered mesostructures inside the columnar alumina pores and the mesostructure can be regulated by humidity and by the silica-to-surfactant ratio as mentioned in section 3. However, the most critical disadvantage of these methods is the formation of mesoporous silica not only inside the columnar alumina pore but also at the membrane surface. $^{20,28}$ The use of the hybrid mesoporous membrane for nanofluidic systems is based on mass transport inside the silicananochannels formed inside the columnar alumina pores. The mesoporous silica formed at the membrane surface would 


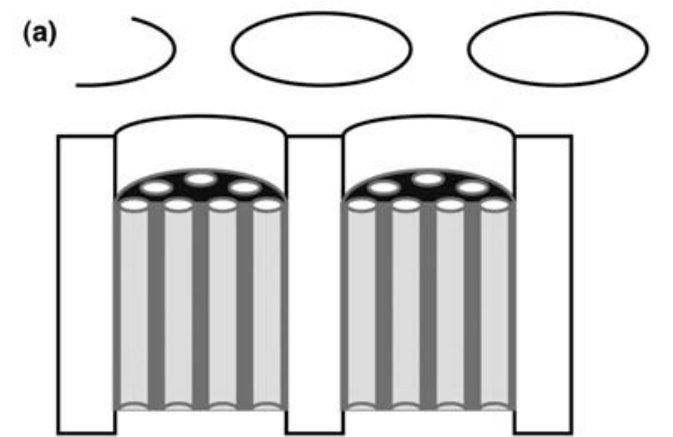

(b)

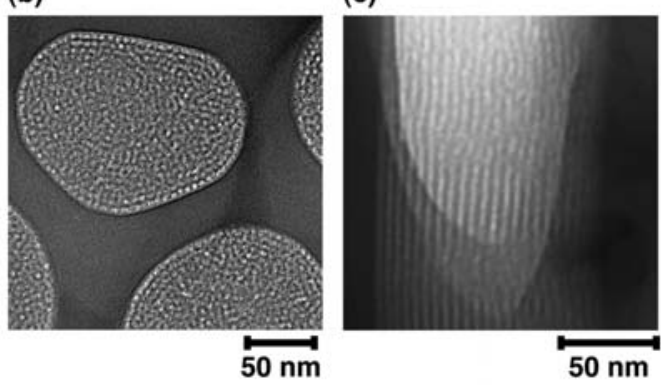

Fig. 3 (a) Schematic illustration of hybrid mesoporous membranes with columnar mesoporous silica composed of 1D silicananochannels. ${ }^{18}$ TEM top-view (b) and cross-sectional view (c) of the columnar mesoporous silica prepared by using $\mathrm{CTAB}$ as the structural directing reagent.

prevent mass transport through the silica-nanochannels inside the columnar alumina pores. Therefore, the hybrid mesoporous membrane fabricated by the aspiration method seems to be applicable to the nanofluidic systems.

\section{Mesostructures}

In general, the mesostructure of mesoporous silica can be regulated by choosing a structural directing reagent (surfactant) and the composition of the precursor solution, and by controlling the temperature and humidity. Until now, the surfactants used for the fabrication of hybrid mesoporous membranes include cetyltrimethylammonium bromide (CTAB), ${ }^{18,26,28}$ decaethylene glycol hexadecyl ether (Brij 56), ${ }^{27,28}$ and poly(ethylene oxide)poly(propylene oxide)-poly(ethylene oxide) (PEO-PPO-PEO) triblock copolymers such as $\mathrm{PEO}_{20} \mathrm{PPO}_{70} \mathrm{PEO}_{20}$ (Pluronic $\mathrm{P} 123)^{20,21,24-26,28-30}$ and $\mathrm{PEO}_{106} \mathrm{PPO}_{70} \mathrm{PEO}_{106}$ (Pluronic F127). 19,22,23,30 Among these surfactants, CTAB has been well known to form mesoporous silica composed of 1D silicananochannels, ${ }^{2}$ and the first achievement of hybrid mesoporous membrane with 1D silica-nanochannels was reported by using CTAB as the structural directing reagent. ${ }^{18}$ Figures 3 (b) and (c) show transmission electron microscopy (TEM) images of the CTAB-based hybrid mesoporous membrane. The silicananochannels are oriented along the columnar alumina pores as schematically shown in Fig. 3(a).

In contrast to $\mathrm{CTAB}$, the uses of other surfactants (block copolymers such as Brij 56, P123, and F127) sometimes cause formation of not only $1 \mathrm{D}$ silica-nanochannels but also other 3D mesostructures. Typical TEM images of the columnar mesoporous silica synthesized by using P123 are shown in Fig. 4. ${ }^{30} \mathrm{Wu}$ et al. $^{25}$ examined the mesostructure of mesoporous silica prepared by using P123 as a structural directing reagent. In this study, they found formation of unprecedented 3D (a)

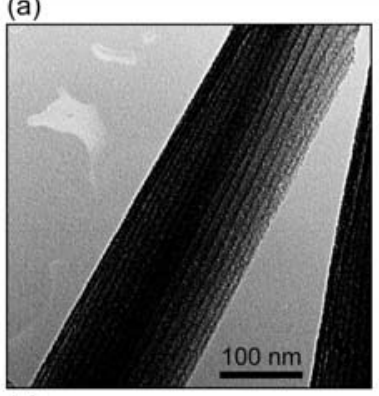

(c)

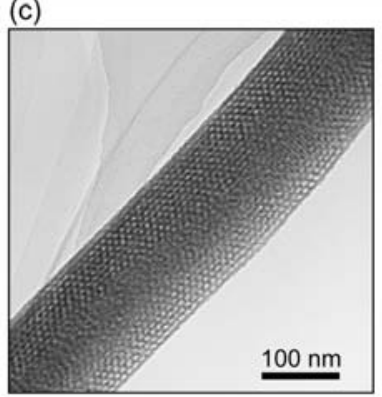

(b)

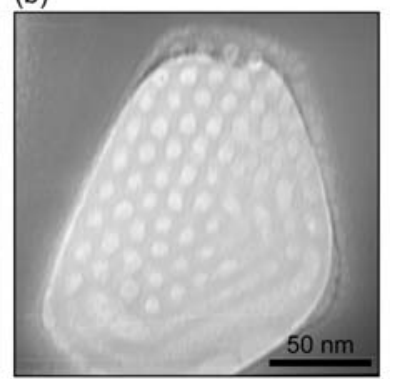

(d)

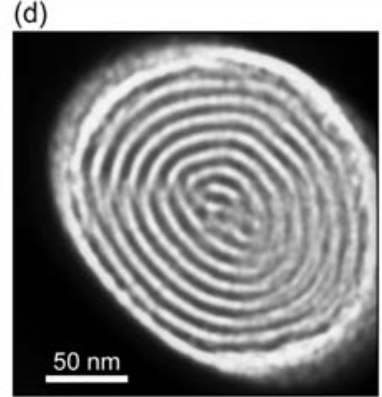

Fig. 4 TEM images of columnar mesoporous silica formed inside the columnar alumina pore: ${ }^{30}$ TEM top (a) and cross-sectional (b) views of columnar mesoporous silica with $1 \mathrm{D}$ silica-nanochannels, TEM top (c) and cross-sectional (d) views of columnar mesoporous silica with $3 \mathrm{D}$ mesostructure.

mesostructures with chiral mesopores such as single- and double-helical geometries, and transition in the mesostructure from a coiled cylindrical to a spherical cage-like geometry with decreasing the diameter of the columnar alumina pore (Fig. 5). The columnar mesoporous silica with similar 3D mesostructures shown in Figs. 4(c) and (d) were also reported for the hybrid mesoporous membrane by using Brij56 and F127. ${ }^{19,28,30}$ Platschek et al..$^{28}$ further examined the 3D mesostructures by TEM and 2D small-angle X-ray scattering (SAXS) measurements.

Although the use of block copolymer surfactants sometimes results in formation of columnar mesoporous silica with 3D mesostructure, the regulation from the $3 \mathrm{D}$ to $1 \mathrm{D}$ mesostructure could be achieved by optimizing the fabrication conditions. Yao et al. ${ }^{21}$ examined the effects of humidity for the mesostructure of the columnar mesoporous silica formed by immersing a PAA membrane in a precursor solution containing P123. Their results showed that the 1D silica-nanochannels could be formed under a low humidity environment, whereas the high humidity environment resulted in the 3D mesostructure. This result was explained by the gelation rate; since the gelation process was slower in lower humidity environment, the slow gelation process would be due to the formation of 1D silicananochannels. Platschek et al. ${ }^{28}$ reported that the $1 \mathrm{D}$ silicananochannels could be formed in lower humidity environments and with lower silica to surfactant ratios when block copolymer surfactants (P123 and Brij56) were used as a structural directing reagent.

In the studies on regulation of mesostructure, the mesoporous silica was formed inside the columnar alumina pores by the soaking (Fig. 2(a)) or EISA (Fig. 2(b)) method; attention was given to the control of temperature and humidity during the growth of silica-surfactant nanocomposites. In contrast, the control of temperature and humidity is difficult for the aspiration method. However, the regulation of mesostructure could be achieved by controlling the aging condition of a precursor 


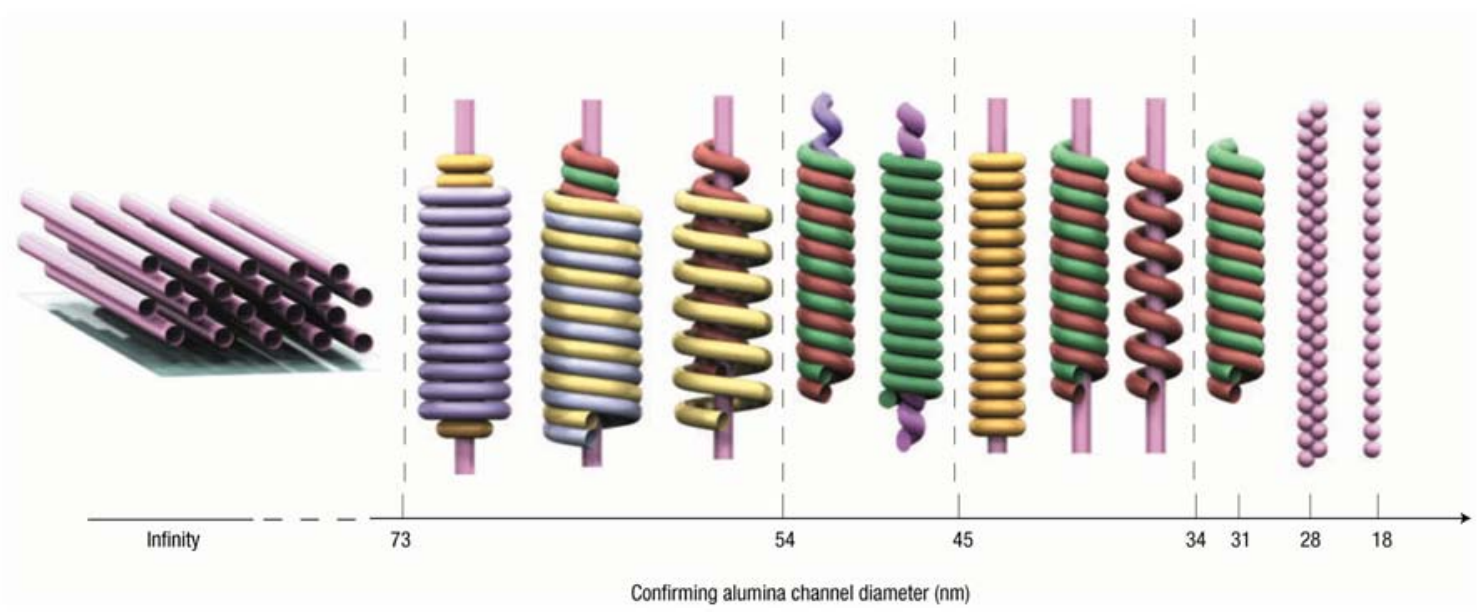

Fig. 5 Summary of mesostructures formed inside the alumna pores with different pore diameter. Reprinted with permission from Ref. 25. Copyright 2005 Nature Publishing Group.

solution. ${ }^{30}$ In this study, a series of precursor solutions containing P123 were prepared by changing the aging temperatures and times, and were applied for the formation of the columnar mesoporous silica by the aspiration method. Results showed that predominant formation of the 1D silicananochannels (Figs. 4(a) and (b)) could be achieved by increasing the aging temperature $\left(0\right.$ to $\left.60^{\circ} \mathrm{C}\right)$ and time (1 to 12 h). In addition, the optimum ratio of silica to surfactant in the precursor solution was also confirmed.

Until now, the 1D hybrid mesoporous membrane, which is composed of columnar mesoporous silica with 1D silicananochannels inside the alumina pores, has been fabricated by using CTAB, Brij56 and P123 as the structural directing reagents. The reported structural parameters are as follows: 3.4 $\mathrm{nm}(\mathrm{CTAB}),{ }^{16} 6 \mathrm{~nm}(\mathrm{P} 123),{ }^{20}$ and $8 \mathrm{~nm}(\mathrm{P} 123)^{30}$ as channel diameter, and $4.3 \mathrm{~nm}$ (CTAB), ${ }^{28} 6.2 \mathrm{~nm}$ (Brij56), ${ }^{28} 8.6 \mathrm{~nm}$ $(\mathrm{P} 123),{ }^{21}$ and $11 \mathrm{~nm}(\mathrm{P} 123)^{28}$ as $d_{(100)}$-spacing (channel to channel distance). Considering studies on mesoporous silica powders and thin films, ${ }^{31}$ further regulation of the channel diameter can be expected for the hybrid mesoporous membrane by choosing other surfactants as the structural directing reagents in order to tune channel diameter from a few to tens of nanometers. In addition, the formation of mesoporous silica inside micropores such as the alumina pore sometimes results in unprecedented mesostructures that had not been found for the mesoporous silica powders and thin films..$^{25,26}$ Thus, further studies on tuning of channel diameter of silica-nanochannel within the hybrid mesoporous membrane are necessary.

\section{Analytical Applications}

Among analytical applications of the hybrid mesoporous membrane, the size exclusive separation was firstly demonstrated. The hybrid mesoporous membrane with 1D silica-nanochannels (channel diameter $=3.4 \mathrm{~nm}$ ) was mounted in a U-tube permeation cell, and mass transports of molecules were examined (Fig. 6). ${ }^{16}$ The results showed selective transport of molecules depending on the molecular size; relatively small molecules, such as rhodamine B (molecular size, $c a .1 .0 \mathrm{~nm}$ ) and vitamin B12 (ca.2.4 nm), were transported but not large molecules, such as myoglobin $(\mathrm{ca} .4 .0 \mathrm{~nm})$ and bovine serum albumin (ca.7.2 nm) (Fig. 6b). The transport through the membrane was completely rejected for molecules with larger
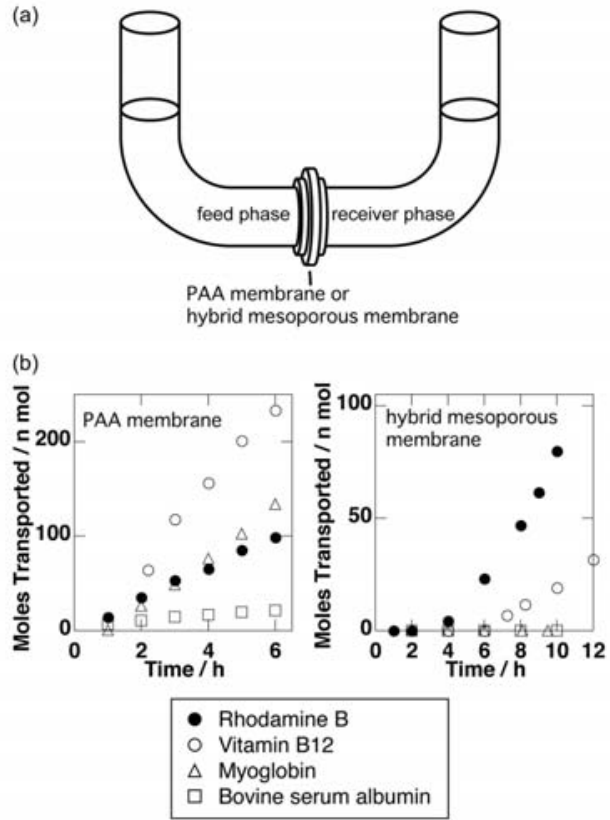

Fig. 6 (a) Schematic illustration of the membrane transport experiment, and (b) observed time-dependent transport of molecules through the PAA membrane and the hybrid mesoporous membrane. ${ }^{18}$

molecular size than the channel diameter $(3.4 \pm 0.2 \mathrm{~nm})$. Accordingly, we concluded that the large molecules could not permeate into the silica-nanochannels formed inside the columnar alumina pores, and that the silica-nanochannels could work to separate molecules with a capability of nm-order size exclusive characteristics.

Yoo et al. ${ }^{27}$ examined the transport of several gases (helium, nitrogen, and propane) across the hybrid mesoporous membrane that had hexagonal and cubic mesostructure with $c a .5 \mathrm{~nm}$ in pore diameter. The hybrid mesoporous membrane gave the higher $\mathrm{He} / \mathrm{N}_{2}$ permselectivity than the commercial PAA membrane ( $5 \mathrm{~nm}$ nominal pore size) which contained pinhole defects. They suggested that the higher permselectivity could be ascribed to the lack of pinhole defects in their hybrid mesoporous membrane.

There was an attempt to incorporate the hybrid mesoporous 
(a)

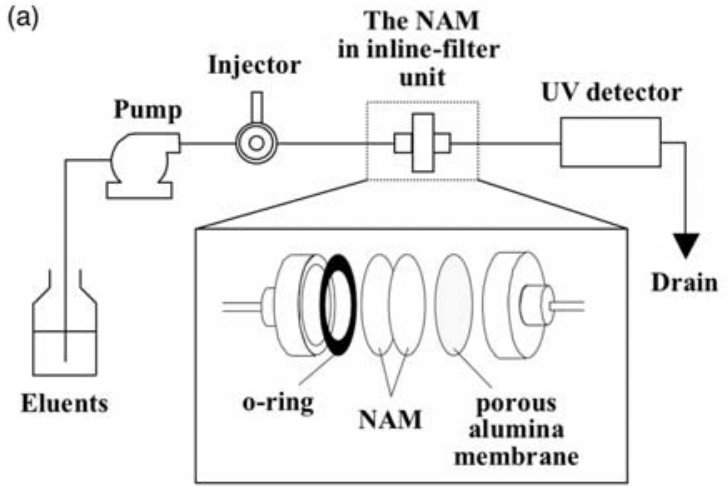

(b)

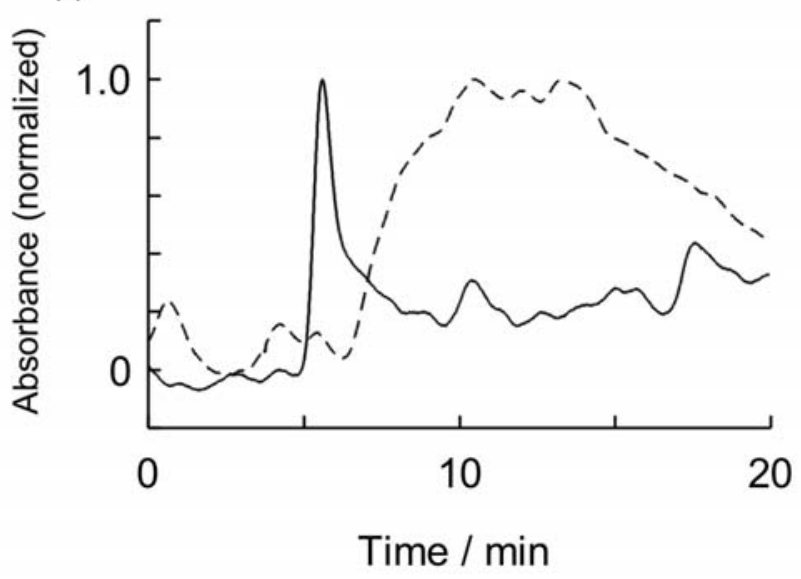

Fig. 7 (a) Schematic illustration of a HPLC system using the hybrid mesoporous membranes, and (b) observed chromatograms of toluene (solid line) and phenol (dashed line). ${ }^{32}$

membrane with 1D silica-nanochannels (channel diameter $=3.4$ $\mathrm{nm}$ ) into a HPLC system as shown in Fig. 7(a). ${ }^{32}$ The observed chromatograms showed that the retention time of phenol was larger than that of toluene (Fig. 7(b)). This result implies that the hybrid mesoporous membrane can be applicable to a chromatographic system. However, for the practical use of the hybrid mesoporous membrane, further improvement in peak separation would be necessary.

In addition to the separation science, other promising analytical applications of the hybrid mesoporous membrane include enzymatic biosensors. Since immobilization of enzymes within mesoporous silica increases the stability of enzymes, the hybrid mesoporous membrane will be available for membranebased enzymatic biosensor chips. Furthermore, metallic or semiconductor nanostructures can be synthesized within 1D (or 3D) silica nanochannels of a hybrid mesoporous membrane. Such nanostructures have a potential use for various sensors by utilizing their inherent optical and electrical properties of those nanostructures. ${ }^{22,25,29,33,34}$

Although the hybrid mesoporous membrane with 1D silicananochannels can be applicable to various fields of analytical chemistry, several problems to be overcome still remain in order to develop innovative separation systems based on the hybrid mesoporous membranes. Since the hybrid mesoporous membrane with long silica-nanochannels is advantageous to chromatographic application, fabrication of long columnar mesoporous silica inside columnar alumina pore is attractive. Most studies on the hybrid mesoporous membrane have dealt with commercially available PAA membranes with $60 \mu \mathrm{m}$ in thickness. It might be desirable to develop a method for fabrication of PAA membranes with more than $60 \mu \mathrm{m}$ in thickness to obtain long columnar mesoporous silica within the thick PAA membrane. A functionalization of the inner surfaces of silica-nanochannels is also important for regulation of mass transport through the silica-nanochannels. In addition, a quantitative characterization of chemical processes that occur inside the silica-nanochannels is important to design separation systems based on the hybrid mesoporous membrane. ${ }^{35-37}$ In particular, essential phenomena to be solved are adsorption or distribution at the silica-nanochannel, viscosity of a solvent confined in the silica-nanochannel, and diffusivity of a solute.

\section{Summary}

We have reviewed fabrication of the hybrid mesoporous membranes and their structural features as well as analytical applications. The growth of surfactant-templated mesoporous silica inside a columnar alumina pore sometimes results in formation of 1D alignment of the surfactant-templated silicananochannels, and this channel orientation allows the use of silica-nanochannels for a nanofluidic systems. Since the first report of the hybrid mesoporous membrane with 1D silicananochannels in 2004, extensive studies have been reported to regulate the mesostructure of mesoporous silica in the hybrid mesoporous membranes, and the hybrid mesoporous membranes with 1D silica-nanochannels have been successfully synthesized. In addition, several studies have reported success in potential uses of the hybrid mesoporous membrane in separation science. We believe that further studies on the hybrid mesoporous silica will lead to the development of innovative analytical systems by utilizing mass transport through the 1D silica-nanochannels.

\section{References}

1. C. T. Kresge, M. E. Leonowicz, W. J. Roth, J. C. Vartuli, and J. S. Beck, Nature, 1992, 359, 710.

2. J. S. Beck, J. C. Vartuli, W. J. Roth, M. E. Leonowicz, C. T. Kresge, K. D. Schmitt, C. T.-W. Chu, D. H. Olson, E. W. Sheppard, S. B. McCullen, J. B. Higgins, and J. L. Schlenker, J. Am. Chem. Soc., 1992, 114, 10834.

3. T. Yanagisawa, T. Shimizu, K. Kuroda, and C. Kato, Bull. Chem. Soc. Jpn., 1990, 63, 988.

4. S. Inagaki, Y. Fukushima, and K. Kuroda, J. Chem. Soc. Chem. Commun., 1993, 680.

5. X. Feng, G. E. Fryxell, L.-Q. Wang, A. Y. Kim, J. Liu, and K. M. Kemner, Science, 1997, 276, 923.

6. H. Yoshitake, New J. Chem., 2005, 29, 1107.

7. V. S.-Y. Lin, C.-Y. Lai, J. Huang, S.-A. Song, and S. Xu, J. Am. Chem. Soc., 2001, 123, 11510.

8. H. H. P. Yiu, C. H. Botting, N. P. Botting, and P. A. Wright, Phys. Chem. Chem. Phys., 2001, 3, 2983.

9. M. Raimondo, G. Perez, M. Sinibaldi, A. De Stefanis, and A. A. G. Tomlinson, Chem. Commun., 1997, 1343.

10. M. Grün, A. A. Kurganov, S. Schacht, F. Schüth, and K. K. Unger, J. Chromatogr., A, 1996, 740, 1.

11. T. Nassivera, A. G. Eklund, and C. C. Landry, J. Chromatogr., A, 2002, 973, 97.

12. C. Boissiére, M. Kümmel, M. Persin, A. Larbot, and E. Prouzet, Adv. Funct. Mater., 2001, 11, 129.

13. T. Yamada, H.-S. Zhou, H. Uchida, M. Tomita, Y. Ueno, T. Ichino, I. Honma, K. Asai, and T. Katsube, Adv. Mater. 2002, 14, 812 . 
14. N. Liu, D. R. Dunphy, P. Atanassov, S. D. Bunge, Z. Chen, G. P. López, T. J. Boyle, and C. J. Brinker, Nano Lett., 2004, 4, 551.

15. C. J. Brinker, Y. Lu, A. Sellinger, and H. Fan, Adv. Mater., 1999, 11, 579 .

16. Y. Yamauchi, M. Sawada, T. Noma, H. Ito, S. Furumi, Y. Sakka, and K. Kuroda, J. Mater. Chem., 2005, 15, 1137.

17. H. Masuda, H. Asoh, M. Watanabe, K. Nishio, M. Nakao, and T. Tamamura, Adv. Mater., 2001, 13, 189.

18. A. Yamaguchi, F. Uejo, T. Yoda, T. Uchida, Y. Tanamura, T. Yamashita, and N. Teramae, Nat. Mater., 2004, 3, 337.

19. Z. Yang, Z. Niu, X. Cao, Z. Yang, Y. Lu, Z. Hu, and C. C. Han, Angew. Chem., Int. Ed., 2003, 42, 4201.

20. Q. Lu, F. Gao, S. Komarneni, and T. E. Mallouk, J. Am. Chem. Soc., 2004, 126, 8650.

21. B. Yao, D. Fleming, M. A. Morris, and S. E. Lawrence, Chem. Mater., 2004, 16, 4851.

22. W.-S. Chae, S.-W. Lee, S.-J. Im, S.-W. Moon, W.-C. Zin, J.K. Lee, and Y.-R. Kim, Chem. Commun., 2004, 2554.

23. W.-S. Chae, S.-W. Lee, M.-J. An, K.-H. Choi, S.-W. Moon, W.-C. Zin, J.-S. Jung, and Y.-R. Kim, Chem. Mater, 2005, 17, 2554.

24. K. Jin, B. Yao, and N. Wang, Chem. Phys. Lett., 2005, 409, 172.

25. Y. Wu, G. Cheng, K. Katsov, S. W. Sides, J. Wang, J. Tang, G. H. Fredrickson, M. Moskovits, and G. D. Stucky, Nat. Mater, 2004, 3, 816.

26. A. Y. Ku, S. T. Taylor, and S. M. Loureiro, J. Am. Chem. Soc., 2005, 127, 6934.
27. S. Yoo, D. M. Ford, and D. F. Shantz, Langmuir, 2006, 22, 1839.

28. B. Platschek, N. Petkov, and T. Bein, Angew. Chem., Int. Ed., 2006, 45, 1134.

29. N. Petkov, B. Platschek, M. A. Morris, J. D. Holmes, and T. Bein, Chem. Mater., 2007, 19, 1376.

30. A. Yamaguchi, H. Kaneda, W. Fu, and N. Teramae, $A d v$. Mater, in press.

31. G. J. de A. A. Soler-Illia, C. Sanchez, B. Lebeau, and J. Patarin, Chem. Rev., 102, 4093.

32. T. Yamashita, S. Kodama, M. Ohto, E. Nakayama, S. Hasegawa, N. Takayanagi, T. Kemmei, A. Yamaguchi, N. Teramae, and Y. Saito, Anal. Sci., 2006, 22, 1495.

33. Y. Wu, T. Livneh, Y. X. Zhang, G. Cheng, J. Wang, J. Tang, M. Moskovits, and G. D. Stucky, Nano Lett., 2004, 4, 2337.

34. W.-S. Chae, S.-W. Lee, M.-J. An, K.-H. Choi, S.-W. Moon, W.-C. Zin, J.-S. Jung, and Y.-R. Kim, Chem. Mater, 2005, 17,5651 .

35. Y. Chen, A. Yamaguchi, T. Atou, K. Morita, and N. Teramae, Chem. Lett., 2006, 35, 1352.

36. A. Yamaguchi, J. Watanabe, M. M. Mahmoud, R. Fujiwara, K. Morita, T. Yamashita, Y. Amino, Y. Chen, L. Radhakrishnan, and N. Teramae, Anal. Chim. Acta, 2006, 556, 157.

37. A. Yamaguchi, Y. Amino, K. Shima, S. Suzuki, T. Yamashita, and N. Teramae, J. Phys. Chem. B, 2006, 110, 3910.

38. A. Yamaguchi, T. Yoda, S. Suzuki, K. Morita, and N. Teramae, Anal. Sci., 2006, 22, 1501. 